\title{
NORMAL DEVELOPABLE SURFACES OF A SURFACE ALONG A DIRECTION CURVE
}

\author{
Rashad A. Abdel-Baky ${ }^{1}$, Yasin Unluturk ${ }^{2}$ \\ ${ }^{1}$ Department of Mathematics, Faculty of Science, University of Assiut, \\ Assiut 71516, EGYPT, e-mail: rbaky@live.com \\ ${ }^{2}$ Department of Mathematics, Kırklareli University, 39100, Kırklareli, \\ TURKEY, e-mail: yasinunluturk@klu.edu.tr
}

\begin{abstract}
We construct a developable surface normal to a surface along a curve on the surface. We choose the curve as the normal direction curve on which the new surface is formed in Euclidean space. We obtain some results about the uniqueness and the singularities of such developable surfaces. We also give two invariants of curves on a surface which characterize singularities.

Keywords: Normal developable surfaces, Curves on surfaces, Ruled surfaces, Direction curve, Singularities
\end{abstract}

\section{INTRODUCTION}

The motion of a straight line continuously along a curve generates a surface called ruled surface. In other words, ruled surfaces are one-parameter family of lines. It is a very classical subject which has been studied in differential geometry for two hundred years. Applications of ruled surfaces have been extensively performed to computer-aided geometric design (CAGD), surface design, manufacturing technology, simulation and rigid bodies. A regular ruled surface in Euclidean 3 -space $\mathbb{R}^{3}$ whose Gaussian curvature vanishes is called a developable surface. Developable surfaces as a kind of ruled surfaces are classified into cylinders, cones or tangent surfaces of space curves [1], [3], [10], [11], [12].

Izumiya and Takeuchi, in their survey of ruled surfaces [4], presented original results about curves on ruled surfaces in Euclidean space. They studied curves on ruled surfaces by choosing curves as cylindirical helices and Bertrand curves [5]. In their another paper [6], they defined the notion of slant helices and conical geodesic curves which are generalizations of the notion of helices. They also constructed the tangential Darboux developable of a space curve and examined singularities of this surface. They gave interesting results about a geometric invariant of space curve

2020 Mathematics Subject Classification: 53A04, 53A05, 57R45

Received: 13-09-2019, accepted: 16-06-2020. 
which is closely regarded to singularities of the tangential Darboux developable of the original curve [6].

The motivation of this study is based on the works of Izumiya and Otani [7], and Hananoi and Izumiya [8]. In the first paper [7], the authors constructed osculating developable surface along the curve on the surface by taking a developable surface tangent to a surface along a curve on the surface into consideration. Then they gave some results such as the uniqueness and the singularities of such a surface. In the second work [8], Hananoi and Izumiya studied a developable surface which remains normal to a surface along a curve on ruled surface. They had results such as the uniqueness and the singularities of such developable surfaces. Recently, Markina and Raffaelli examined the same topic in $\mathbb{R}^{m+1}$. Taking a smooth curve $\gamma$ in an $m$-dimensional surface $M$ in $\mathbb{R}^{m+1}$, they gave some results about the existence and uniqueness of a flat surface $H$ having the same field of normal vectors as $M$ along $\gamma[9]$.

The organization of this work is as follows: in Sec. 2, we gave the basic notions and definitions of curve on ruled surfaces and developable surfaces in Euclidean space. In Sec. 3, new developable surfaces which remain normal to the base surface are constructed along a normal direction curve and some results such as invariants of $M_{n}$ characterizing contour generators of $M$, and existence and uniqueness of the surface have been presented for these surfaces. Finally, illustrative examples have been given for the base surface and its normal developable surface.

\section{PRELIMINARIES}

In this section we list some notions, formulas and conclusions for space curves, and ruled surfaces in Euclidean 3 -space $\mathbb{R}^{3}$ which can be found in the textbooks on differential geometry (See for instance Refs. [4], [6], [11]).

Let $M$ be a regular surface in $\mathbb{R}^{3}$ and $\alpha: I \subseteq \mathbb{R} \rightarrow M$ be a unit speed curve. At each point on $\alpha=\alpha(s)$, consider the following three vectors: the unit normal vector $e_{3}(s)$ to the surface, the unit tangent vector $e_{1}=e_{1}(s)$ to the curve and the tangent normal vector $e_{2}=e_{3} \times e_{1}$. The vector $e_{2}$ is tangent to the surface $M$ , but normal to the curve $\alpha=\alpha(s)$. Then we have an orthonormal frame $\left\{e_{1}(s)\right.$, $\left.e_{2}(s), e_{3}(s)\right\}$ along $\alpha$, which is called the Darboux frame along $\alpha=\alpha(s)$. Darboux equations for this frame are given by

$$
\left[\begin{array}{l}
e_{1}^{\prime} \\
e_{2}^{\prime} \\
e_{3}^{\prime}
\end{array}\right]=\left[\begin{array}{lll}
0 & \kappa_{g} & \kappa_{n} \\
-\kappa_{g} & 0 & \tau_{g} \\
-\kappa_{n} & -\tau_{g} & 0
\end{array}\right]\left[\begin{array}{l}
e_{1} \\
e_{2} \\
e_{3}
\end{array}\right]
$$

Here,

$$
\left.\begin{array}{l}
\kappa_{g}(s):=\left\langle e_{1}^{\prime}, e_{2}\right\rangle=\operatorname{det}\left(\alpha^{\prime}, \alpha^{\prime \prime}, e_{3}\right) \\
\kappa_{n}(s):=\left\langle e_{1}^{\prime}, e_{3}\right\rangle=\left\langle\alpha^{\prime \prime}, e_{3}\right\rangle, \\
\tau_{g}(s):=\left\langle e_{2}^{\prime}, e_{3}\right\rangle=\operatorname{det}\left(\alpha^{\prime}, e_{3}, e_{3}^{\prime}\right) .
\end{array}\right\}
$$

In terms of these quantities, the geodesics, asymptotic lines, and line of curvatures on a smooth surface may be characterized, as loci along which $\kappa_{g}(s)=0, \kappa_{n}=0$, and $\tau_{g}(s)=0$, respectively. 
On the other hand, it is known that

$$
\kappa(s)=\sqrt{\kappa_{g}^{2}+\kappa_{n}^{2}}, \quad \text { and } \tau_{g}(s)=\frac{\kappa_{n} \kappa_{g}^{\prime}-\kappa_{g} \kappa_{n}^{\prime}}{\kappa_{n}^{2}+\kappa_{g}^{2}}+\tau(s),
$$

where $\kappa(s)$, and $\tau(s)$ are the curvature and the torsion of $\alpha=\alpha(s)$ as a space curve, respectively [10]. From now on, we shall often not write the parameter $s$ explicitly in our formulae.

2.1. Ruled and developable surfaces. A ruled surface in Euclidean 3-space $\mathbb{R}^{3}$ is a differentiable one-parameter set of straight lines. Such a surface has a parameterization as

$$
\mathbf{P}(s, v)=\alpha(s)+v \mathbf{e}(s), v \in \mathbb{R},
$$

where $\alpha(s)$ is its base curve and $\mathbf{e}(s)$ is the unit vector giving the direction of the straight lines of the surface. The unit normal vector of the ruled surface $\mathbf{P}(s, v)$ at each point is defined by

$$
\mathbf{n}(s, v)=\frac{\mathbf{P}_{s} \times \mathbf{P}_{v}}{\left\|\mathbf{P}_{s} \times \mathbf{P}_{v}\right\|}=\frac{\alpha^{\prime} \times \mathbf{e}+v \mathbf{e}^{\prime} \times \mathbf{e}}{\left\|\alpha^{\prime} \times \mathbf{e}+v \mathbf{e}^{\prime} \times \mathbf{e}\right\|} .
$$

The base curve is not unique, since any curve of the form:

$$
\mathbf{C}(s)=\alpha(s)-\eta(s) \mathbf{e}(s),
$$

may be used as its base curve, $\eta(s)$ is a smooth function. If there exists a common perpendicular to two neighbouring rulings on $\mathbf{P}(s, v)$, then the foot of the common perpendicular on the main ruling is called a central point. The locus of the central points is called the striction curve. In Eq. (6) if

$$
\eta(s)=\frac{\left\langle\alpha^{\prime}(s), \mathbf{e}^{\prime}\right\rangle}{\left\|\mathbf{e}^{\prime}\right\|^{2}} .
$$

then the curve $\mathbf{C}(s)$ is called the striction curve on the ruled surface and it is unique. In the case of $\eta=0$, the base curve is the striction curve. The distribution parameter of $\mathbf{P}(s, v)$ is defined by

$$
\lambda(s)=\frac{\operatorname{det}\left(\alpha^{\prime}, \mathbf{e}, \mathbf{e}^{\prime}\right)}{\left\|\mathbf{e}^{\prime}\right\|^{2}} .
$$

The distribution parameter $\lambda$ is a well-known real integral invariant of a ruled surface and allows further classification of the ruled surface.

Developable surfaces can be briefly introduced as special cases of ruled surfaces. If the ruled surface $\mathbf{P}(s, v)$ is a developable one, then we have

$$
\lambda(s)=0 \Leftrightarrow \operatorname{det}\left(\alpha^{\prime}, \mathbf{e}, \mathbf{e}^{\prime}\right)=0 .
$$

Thus a volume formed by $\alpha^{\prime}$, e and $\mathbf{e}^{\prime}$ vanishes, i.e, they are linearly dependent. This condition (9) is satisfied provided that there are three non-identically vanishing functions $\eta(s), \beta(s)$ and $\gamma(s)$ satisfying

$$
\mu(s) \alpha^{\prime}+\xi(s) \mathbf{e}+\gamma(s) \mathbf{e}^{\prime}=\mathbf{0} .
$$


We must consider the following cases:

Case 1: $\mu=0$

Since $\left\langle\mathbf{e}, \mathbf{e}^{\prime}\right\rangle=0$, it follows immediately that Eq. (7) is only satisfied when $\mathbf{e}$ is a constant vector, i.e., $\mathbf{P}(s, v)$ is a part of a cylinder.

Case 2: $\mu \neq 0$ from Eq. (7) it follows:

$$
\alpha^{\prime}=\zeta(s) \mathbf{e}+v(s) \mathbf{e}^{\prime}
$$

where

$$
\zeta(s)=-\frac{\xi}{\mu}, v(s)=-\frac{\gamma}{\mu} .
$$

Differentiating Eq. (6) and using Eq. (8), we get

$$
\mathbf{C}^{\prime}(s)=\left(\zeta(s)-\eta^{\prime}(s)\right) \mathbf{e}(s)+(v(s)-\eta(s)) \mathbf{e}^{\prime} .
$$

The condition for $\mathbf{C}$ to be striction curve is equivalent to that the vectors $\mathbf{C}^{\prime}$ and $\mathbf{e}^{\prime}$ are perpendicular to each other. Therefore, we conclude that the ruling is parallel to the first derivative of the striction curve, which is also the tangent of the striction curve, i.e.

$$
\mathbf{C}^{\prime}=\left(\zeta(s)-\eta^{\prime}(s)\right) \mathbf{e}(s) .
$$

We must hence consider the following sub-case: $\zeta(s)=\eta^{\prime}(s)$. In this case, Eq. (13) yields $\mathbf{C}=\mathbf{C}_{0}$ is a constant vector. So, $\mathbf{P}(s, v)$ is a part of a cone as

$$
\mathbf{P}(s, v)=\mathbf{C}_{0}+(\eta(s)+v) \mathbf{e}(s), v \in \mathbb{R} .
$$

We now define the notion of contour generators. Let $M$ be an orientable surface and $\mathbf{n}$ a unit normal vector field on $M$. For a unit vector $\mathbf{x}$ in the unit sphere $\mathbb{S}^{2}=\left\{\mathbf{x} \in \mathbb{R}^{3} \mid\|\mathbf{x}\|=1\right\}$, the normal contour generator of the orthogonal projection with the direction $\mathbf{x}$ is defined to be

$$
\{\mathbf{p} \in M \mid\langle\mathbf{n}, \mathbf{x}\rangle=0\} .
$$

Moreover, for a fixed point $\mathbf{c} \in \mathbb{R}^{3}$, the normal contour generators of the central projection with the center $\mathbf{c}$ are defined to be

$$
\{\mathbf{p} \in M \mid\langle\mathbf{n}, \mathbf{p}-\mathbf{c}\rangle=0\} .
$$

For a regular surface, the notion of contour generators plays an important role in the theory of vision [2].

Let $M \subset \mathbb{R}^{3}$ be a surface. It has been defined by Hananoi and Izumiya [8] that a developable surface $M_{n}$ is a normal developable surface of $M$ if $M_{n} \cap M \neq \emptyset$ and the tangent planes $T_{p} M_{n}$ and $T_{p} M$ are orthogonal at any point $p \in M_{n} \cap M$. If $M_{n}$ is a cylinder, $M_{n}$ becomes a normal cylinder of $M$. Also $M_{n}$ is normal cone of $M$ if $M_{n}$ is cone. For a normal developable surface $M_{n}$ of $M$, the intersection $M_{n} \cap M$ is a regular curve. 


\section{MAIN RESULTS}

In this section, we introduce a normal developable surface along the $e_{3}(s)$ direction curve

$$
\beta(s)=\int_{0}^{s} e_{3}(s) d s .
$$

Under the assumption $\left(\tau_{g}(s), \kappa_{g}(s)\right) \neq(0,0)$, one define the following ruled surface

$$
M_{n}: \widetilde{\mathbf{P}}(s, v)=\beta(s)+v \widetilde{\mathbf{e}}(s),
$$

where $v \in \mathbb{R}$, and

$$
\widetilde{\mathbf{e}}(s)=\frac{\tau_{g} e_{1}+\kappa_{g} e_{3}}{\sqrt{\tau_{g}^{2}+\kappa_{g}^{2}}} .
$$

Firstly, differentiating $\widetilde{\mathbf{e}}$, we find

$$
\widetilde{\mathbf{e}}^{\prime}=\left(\kappa_{n}+\frac{\tau_{g} \kappa_{g}^{\prime}-\tau_{g}^{\prime} \kappa_{g}}{\tau_{g}^{2}+\kappa_{g}^{2}}\right)\left(\frac{-\kappa_{g} e_{1}+\tau_{g} e_{3}}{\sqrt{\tau_{g}^{2}+\kappa_{g}^{2}}}\right),
$$

and thus $\lambda(s)=0$. This means that $M_{n}$ is a developable surface. Moreover, we introduce two invariants $\delta(s)$, and $\sigma(s)$ of $M_{n}$ as follows:

$$
\delta(s)=\kappa_{n}+\frac{\tau_{g} \kappa_{g}^{\prime}-\tau_{g}^{\prime} \kappa_{g}}{\tau_{g}^{2}+\kappa_{g}^{2}}, \quad \text { and } \quad \sigma(s)=\frac{\kappa_{g}}{\sqrt{\tau_{g}^{2}+\kappa_{g}^{2}}}-\left(\frac{\tau_{g}}{\delta(s) \sqrt{\tau_{g}^{2}+\kappa_{g}^{2}}}\right)^{\prime},
$$

where $\delta(s) \neq 0$. We can also calculate that

$$
\widetilde{\mathbf{P}}_{s} \times \widetilde{\mathbf{P}}_{v}=\left(v \delta+\frac{\tau_{g}}{\sqrt{\tau_{g}^{2}+\kappa_{g}^{2}}}\right) e_{2}
$$

Hence, the normal vector of $M_{n}$ is orthogonal to the normal vector of $M$. This is the reason why we call $M_{n}$ the normal developable surface of $M$ along $\beta(s)$.

On the other hand, the invariants $\delta(s)$, and $\sigma(s)$ of $M_{n}$ characterize contour generators of $M$ as follows:

Theorem 3.1. Let $M_{n}$ be the normal developable surface of $M$ along $\beta(s)$ expressed by Eq. (17). Then we have the following:

(A) The following are equivalent:(1) $M_{n}$ is a cylinder,(2) $\delta(s)=0$,(3) $\beta=\beta(s)$ is a contour generator with respect to an orthogonal projection.

(B) If $\delta(s) \neq 0$, then the following are equivalent:(1) $M_{n}$ is a cone,(2) $\sigma(s)=0$,(3) $\beta=\beta(s)$ is a contour generator with respect to a central projection.

Proof. (A) From Eq. (18), it is obvious that $M_{n}$ is a cylinder if and only if $\tilde{\mathbf{e}}(s)$ is constant, i.e. $\delta(s)=0$. Therefore, the condition (1) is equivalent to the condition (2). Suppose that the condition (3) holds. Then there exists a fixed vector $\mathbf{x} \in \mathbb{S}^{2}$ such that $\left\langle e_{2}, \mathbf{x}\right\rangle=0$. So there exist $a, b \in \mathbb{R}$ such that $\mathbf{x}=a e_{1}+b e_{3}$. 
Since $\left\langle e_{2}^{\prime}, \mathbf{x}\right\rangle=0$, we have $a \kappa_{g}+b \kappa_{n}=0$, so that we have $\mathbf{x}=\widetilde{\mathbf{e}}(s)$. Namely, the condition (1) holds. Suppose that $\widetilde{\mathbf{e}}(s)$ is constant. Then we choose $\mathbf{x}=\widetilde{\mathbf{e}}(s) \in \mathbb{S}^{2}$. By the definition of $\widetilde{\mathbf{e}}(s)$, we have $\left\langle\mathbf{x}, e_{2}\right\rangle=0$. Thus the condition (1) implies the condition (3).

(B) The condition (1) means that the singular value set of $M_{n}$ is a constant vector. Thus, in view of Eqs. (6), (7), and from Eq. (19), we have

$$
\mathbf{C}^{\prime}(s)=\left(\frac{\kappa_{g}}{\sqrt{\tau_{g}^{2}+\kappa_{g}^{2}}}-\left(\frac{1}{\delta(s)} \frac{\tau_{g}}{\sqrt{\tau_{g}^{2}+\kappa_{g}^{2}}}\right)^{\prime}\right) \widetilde{\mathbf{e}}(s)=\sigma(s) \widetilde{\mathbf{e}}(s) .
$$

Then $M_{n}$ is a cone if and only if $\sigma(s)=0$. It follows that (1) and (2) are equivalent. By the definition of the the central projection, it can be said that there exists a fixed point $\mathbf{c} \in \mathbb{R}^{3}$ such that $\left\langle e_{2}, \beta-\mathbf{c}\right\rangle=0$. If (1) holds, then $\mathbf{C}(s)$ is constant. For the constant point $\mathbf{c}=\mathbf{C}(s)$, we have

$$
\left\langle e_{2}, \beta-\mathbf{c}\right\rangle=\left\langle e_{2}, \beta-\mathbf{C}\right\rangle=\left\langle e_{2}, \frac{\left\langle\beta^{\prime}, \widetilde{\mathbf{e}}^{\prime}>\right.}{\left\|\widetilde{\mathbf{e}}^{\prime}\right\|^{2}} \mathbf{e}\right\rangle=\left\langle e_{2}, \widetilde{\mathbf{e}}\right\rangle=0 .
$$

This means that (3) holds. For the converse, by (3), there exists a fixed point $\mathbf{c} \in \mathbb{R}^{3}$ such that $\left\langle e_{2}, \beta-\mathbf{c}\right\rangle=0$. Taking the derivative of the both side, we have

$$
0=\left\langle e_{2}, \beta-\mathbf{c}\right\rangle^{\prime}=\left\langle-\kappa_{g} e_{1}+\tau_{g} e_{3}, \beta-\mathbf{c}\right\rangle,
$$

so we may write $\beta-\mathbf{c}=f(s) \widetilde{\mathbf{e}}(s)$, where $f(s)$ is a differentiable function. Taking the derivative again, we have:

$$
0=\left\langle e_{2}, \beta-\mathbf{c}\right\rangle^{\prime \prime}=\left\langle-\kappa_{g} e_{1}+\tau_{g} e_{3}, e_{3}\right\rangle+\left\langle\left(-\kappa_{g} e_{1}+\tau_{g} e_{3}\right)^{\prime}, \beta-\mathbf{c}\right\rangle,
$$

or equivaently,

$$
0=\left\langle e_{2}, \beta-\mathbf{c}\right\rangle^{\prime \prime}=\tau_{g}-f \delta \sqrt{\tau_{g}^{2}+\kappa_{g}^{2}}
$$

It follows that

$$
\mathbf{c}=\beta(s)-f(s) \widetilde{\mathbf{e}}(s)=\beta-\frac{\left\langle\beta^{\prime}, \widetilde{\mathbf{e}}^{\prime}\right\rangle}{\left\|\widetilde{\mathbf{e}}^{\prime}\right\|^{2}} \widetilde{\mathbf{e}}(s)=\mathbf{C}(s) .
$$

Therefore, $\mathbf{C}(s)$ is constant, so that (1) holds $\square$.

Theorem 3.2. (Existence and uniqueness). Let $M \subset \mathbb{R}^{3}$ be a regular surface and $\beta: I \rightarrow M \subset \mathbb{R}^{3}$ be a unit-speed curve given by $\beta=\int e_{3}(s) d s$ with $\kappa_{g}^{2}+\tau_{g}^{2} \neq 0$. Then there exists a unique developable surface which is normal to the surface $M$ along the curve $\beta$.

Proof. For the existence, we have the normal developable surface along $\beta=\beta(s)$ represented by Eq. (17). On the other hand, since $M_{n}$ is a ruled surface, we assume that

$$
M_{n}: \widetilde{\mathbf{P}}(s, v)=\beta(s)+v \zeta(s),
$$


where $v \in \mathbb{R}$,

$$
\zeta(s)=\zeta_{1}(s) e_{1}+\zeta_{2}(s) e_{2}+\zeta_{3}(s) e_{3}, \zeta^{\prime}(s) \neq \mathbf{0} .
$$

It can be immediately seen from Eqs. (1) and (21) that $M_{n}$ is developable if and only if

$$
\operatorname{det}\left(\beta^{\prime}, \zeta, \zeta^{\prime}\right)=0 \Leftrightarrow-\zeta_{2} \zeta_{1}^{\prime}+\zeta_{1} \zeta_{2}^{\prime}-\zeta_{3}\left(\zeta_{1} \tau_{g}+\zeta_{2} \kappa_{n}\right)+\kappa_{g}\left(\zeta_{2}^{2}+\zeta_{1}^{2}\right)=0 .
$$

On the other hand, since $M_{n}$ is a developable surface which is normal developable surface along $\beta=\beta(s)$, we have

$$
\left(\widetilde{\mathbf{P}}_{s} \times \widetilde{\mathbf{P}}_{v}\right)(s, v)=\psi(s, v) e_{2} .
$$

Also, the normal vector $\widetilde{\mathbf{P}}_{s} \times \widetilde{\mathbf{P}}_{v}$ at the point $(s, 0)$ is

$$
\left(\widetilde{\mathbf{P}}_{s} \times \widetilde{\mathbf{P}}_{v}\right)(s, 0)=\zeta_{1} e_{2}-\zeta_{2} e_{1} .
$$

By means of Eqs. (23) and (24) we find:

$$
\zeta_{2}=0, \quad \text { and } \quad \zeta_{1}=\psi(s, 0),
$$

which follows from Eq. (22) that

$$
\zeta_{1}\left(\zeta_{1} \kappa_{g}-\zeta_{3} \tau_{g}\right)=0 .
$$

If $(s, 0)$ is a regular point (i.e., $\psi(s, 0) \neq 0$ ), then $\zeta_{1}(s) \neq 0$. Thus, we have

$$
\zeta_{3}=\frac{\kappa_{g}}{\tau_{g}} \zeta_{1}, \text { with } \tau_{g} \neq 0 \text {. }
$$

Therefore, we obtain

$$
\zeta(s)=\zeta_{1} e_{1}+\frac{\kappa_{g}}{\tau_{g}} \zeta_{1} e_{3}=\frac{\zeta_{1}}{\cos \varphi} \widetilde{\mathbf{e}}(s),
$$

where $\varphi \neq \frac{\pi}{2}$. It follows that $\zeta(s)$ is equal to the direction of $\tilde{\mathbf{e}}(s)$. If $\kappa_{g} \neq 0$ (i.e., $\varphi \neq 0$ ), we have the same result as the above case. On the other hand, suppose that $M_{n}$ has a singular point at $\left(s_{0}, 0\right)$. Then $\psi\left(s_{0}, 0\right)=\zeta_{2}\left(s_{0}\right)=\zeta_{1}\left(s_{0}\right)=0$, and we have $\zeta\left(s_{0}\right)=\zeta_{3}\left(s_{0}\right) e_{3}\left(s_{0}\right)$. If the singular point $\beta\left(s_{0}\right)$ is in the closure of the set of points where the normal developable surface along $\beta(s)$ is regular, then there exists a point $\beta(s)$ in any neighborhood of $\beta\left(s_{0}\right)$ such that the uniqueness of the normal developable surface holds at $\beta(s)$. Passing to the limit $s \rightarrow s_{0}$, uniqueness of the normal developable surface also holds at $s_{0}$. Suppose that there exists an open interval $J \subseteq I$ such that $M_{n}$ is singular at $\beta(s)$ for any $s \in J$. Then $\widetilde{\mathbf{P}}(s, v)=\beta(s)+v \zeta_{3}(s) e_{3}(s)$ for any $s \in J$. This means that $\zeta_{2}(s)=\zeta_{1}(s)=0$ for $s \in J$. It follows that

$$
\left(\widetilde{\mathbf{P}}_{s} \times \widetilde{\mathbf{P}}_{v}\right)(s, v)=v \zeta_{3}^{2}\left(\kappa_{n} e_{2}-v \tau_{g} e_{1}\right) .
$$

Thus the above vector is directed to $e_{2}$, i.e. $\widetilde{\mathbf{P}}_{s} \times \widetilde{\mathbf{P}}_{v} \| e_{2}(s)$ if and only if $\tau_{g}=0$ and $\kappa_{n} \neq 0$ for any $s \in J$. In this case, $\mathbf{e}(s)= \pm e_{1}$. This means that uniqueness holds $\square$.

As a result the following corollaries can be given: 
Corollary 3.3. The normal developable surface represented by Eq. (17) is a noncylindrical ruled surface if and only if $\delta(s) \neq 0$.

Proof. It is a straighforward result from the definition of non-cylindirical ruled surface.

Corollary 3.4. The normal developable surface $M_{n}$ represented by Eq. (17) is a tangential developable if and only if $\delta(s) \neq 0$, and $\sigma(s) \neq 0$.

Proof. According to the proof of Theorem 3.1, when $\delta(s) \neq 0$, and $\sigma(s) \neq 0$, we have $\widetilde{\mathbf{e}}^{\prime} \neq \mathbf{0}$, and $\mathbf{C}^{\prime} \neq \mathbf{0}$. Since $\operatorname{det}\left(\beta^{\prime}, \tilde{\mathbf{e}}, \tilde{\mathbf{e}}^{\prime}\right)=0,<\mathbf{C}^{\prime}, \tilde{\mathbf{e}}^{\prime}>=0$ and $<\tilde{\mathbf{e}}, \tilde{\mathbf{e}}^{\prime}>=0$, we can get $\mathbf{C}^{\prime} \| \tilde{\mathbf{e}}$. This means the surface $M_{n}$ is a tangent surface $\square$.

3.1. Special curves on a surface. In this subsection we consider special curves in the following:

(1) If $\tau_{g}=0$, then $\alpha$ is a line of curvature, and

$$
M_{n}: \widetilde{\mathbf{P}}(s, v)=\beta(s)+v e_{3}(s), v \in \mathbb{R}
$$

which is known as the tangent surface of $\beta(s)$. We can give the following corollary for the surface given by (29).

Corollary 3.5. Let $M_{n}$ be the normal developable surface expressed by Eq. (29) with $\tau_{g}=0$. Then we have the following:

(1) $M_{n}$ is non-singular at points $\beta\left(s_{0}\right)$ if and only if $v_{0} \neq 0$.

(2) $M_{n}$ is locally diffeomorphic to Cuspidal edge at points $\widetilde{\mathbf{P}}\left(s_{0}, v_{0}\right)$ if and only if $v_{0}=-c \kappa_{n}^{-1}\left(s_{0}\right) \neq 0, c \neq 0$, and $\kappa_{n}^{\prime}\left(s_{0}\right) \neq 0$.

(3) $M_{n}$ is locally diffeomorphic to Swallowtail $S W$ at points $\widetilde{\mathbf{P}}\left(s_{0}, v_{0}\right)$ if and only if $v_{0}=-c \kappa_{n}^{-1}\left(s_{0}\right) \neq 0, c \neq 0, \kappa_{n}^{\prime}\left(s_{0}\right)=0$, and $\left(\kappa_{n}^{-1}\right)^{\prime \prime}\left(s_{0}\right) \neq 0$.

Proof. Singularities of the normal developable surface expressed by Eq. (29) are determined

$$
\widetilde{\mathbf{P}}_{s} \times \widetilde{\mathbf{P}}_{v}=v \kappa_{n} e_{2} .
$$

Therefore, $\widetilde{\mathbf{P}}\left(s_{0}, v_{0}\right)$ is non-singular if and only if $\widetilde{\mathbf{P}}_{s} \times \widetilde{\mathbf{P}}_{v} \neq \mathbf{0}$. This condition is equivalent to $v_{0}=-c \kappa_{n}^{-1}\left(s_{0}\right), c \neq 0$. This completes the proof of assertion (1). From the Theorem 3.3 of the paper [6], we know that if there exists a parameter $s_{0}$ such that

$$
\left.v_{0}=-c \kappa_{n}^{-1}\left(s_{0}\right), c \neq 0, \quad \text { and } \quad v_{0}^{\prime}=\frac{c \kappa_{n}^{\prime}\left(s_{0}\right)}{\kappa_{n}^{2}\left(s_{0}\right)} \neq 0 \text { (i.e. } \kappa_{n}^{\prime} \neq 0\right),
$$

then $M_{n}$ is locally diffeomorphic to Cuspidal edge at $\widetilde{\mathbf{P}}\left(s_{0}, v_{0}\right)$. This completes the proof of assertion (2). Again from Theorem 3.3 of [6], we know that if there exists a parameter $s_{0}$ such that

$$
v_{0}=-c \kappa_{n}^{-1}\left(s_{0}\right), c \neq 0, \quad v_{0}^{\prime}=\frac{c \kappa_{n}^{\prime}\left(s_{0}\right)}{\kappa_{n}^{2}\left(s_{0}\right)}=0, \quad \text { and } \quad\left(\kappa_{n}^{-1}\right)^{\prime \prime}\left(s_{0}\right) \neq 0,
$$

then $M_{n}$ is locally diffeomorphic to Swallowtail SW at points $\widetilde{\mathbf{P}}\left(s_{0}, v_{0}\right)$. This completes the proof of assertion (3) $\bullet$. 
(2). If $\kappa_{g}=0$, then $\alpha$ is a geodesic curve, and

$$
M_{n}: \widetilde{\mathbf{P}}(s, v)=\beta(s)+v e_{1}(s), v \in \mathbb{R}
$$

We can give the following corollary for the surface given by (31).

Corollary 3.6. Let $M_{n}$ be the normal developable surface expressed by Eq. (31) with $\kappa_{g}=0$. Then we have the following:

(1) $M_{n}$ is non-singular at points $\beta\left(s_{0}\right)$ if and only if $v_{0} \neq 0$.

(2) $M_{n}$ is locally diffeomorphic to Cuspidal edge at points $\widetilde{\mathbf{P}}\left(s_{0}, v_{0}\right)$ if and only if $v_{0}=-\kappa_{n}^{-1}\left(s_{0}\right) \neq 0$, and $\kappa_{n}^{\prime}\left(s_{0}\right) \neq 0$.

(3) $M_{n}$ is locally diffeomorphic to Swallowtail $S W$ at points $\widetilde{\mathbf{P}}\left(s_{0}, v_{0}\right)$ if and only if $v_{0}=-\kappa_{n}^{-1}\left(s_{0}\right) \neq 0, \kappa_{n}^{\prime}\left(s_{0}\right)=0$, and $\left(\kappa_{n}^{-1}\right)^{\prime \prime}\left(s_{0}\right) \neq 0$.

Proof. Singularities of the normal developable surface expressed by Eq. (31) are determined

$$
\widetilde{\mathbf{P}}_{s} \times \widetilde{\mathbf{P}}_{v}=\left(v \kappa_{n}+1\right) e_{2} .
$$

Therefore, $\widetilde{\mathbf{P}}\left(s_{0}, v_{0}\right)$ is non-singular if and only if $\widetilde{\mathbf{P}}_{s} \times \widetilde{\mathbf{P}}_{v} \neq \mathbf{0}$. This condition is equivalent to $v_{0}=-\kappa_{n}^{-1}\left(s_{0}\right)$. This completes the proof of assertion (1). From the Theorem 3.3 of the study [6], we know that if there exists a parameter $s_{0}$ such that

$$
v_{0}=-\kappa_{n}^{-1}\left(s_{0}\right), \quad \text { and } \quad v_{0}^{\prime}=\frac{\kappa_{n}^{\prime}\left(s_{0}\right)}{\kappa_{n}^{2}\left(s_{0}\right)} \neq 0\left(\text { i.e. } \kappa_{n}^{\prime} \neq 0\right) \text {, }
$$

then $M_{n}$ is locally diffeomorphic to Cuspidal edge at $\widetilde{\mathbf{P}}\left(s_{0}, v_{0}\right)$. This completes the proof of assertion (2). Again from Theorem 3.3 of [6], we know that if there exists a parameter $s_{0}$ such that

$$
v_{0}=-\kappa_{n}^{-1}\left(s_{0}\right), \quad v_{0}^{\prime}=\frac{\kappa_{n}^{\prime}\left(s_{0}\right)}{\kappa_{n}^{2}\left(s_{0}\right)}=0, \quad \text { and } \quad\left(\kappa_{n}^{-1}\right)^{\prime \prime}\left(s_{0}\right) \neq 0
$$

then $M_{n}$ is locally diffeomorphic to Swallowtail SW at points $\widetilde{\mathbf{P}}\left(s_{0}, v_{0}\right)$. This completes the proof of assertion (3) $\bullet$.

3.2. Examples. We close this section with some examples:

Example 3.7. Let the base surface $M$ be given as the following parameterization:

$$
\mathbf{P}(s, v)=\left(\frac{1}{\sqrt{2}} \cos s, \frac{1}{\sqrt{2}} \sin s, \frac{s}{\sqrt{2}}-2 v\right) .
$$




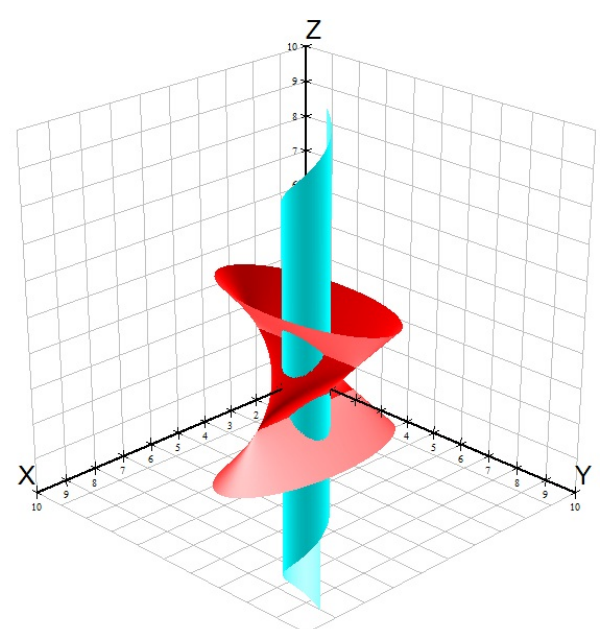

Fig. 1a. The base surface and its normal developable surface

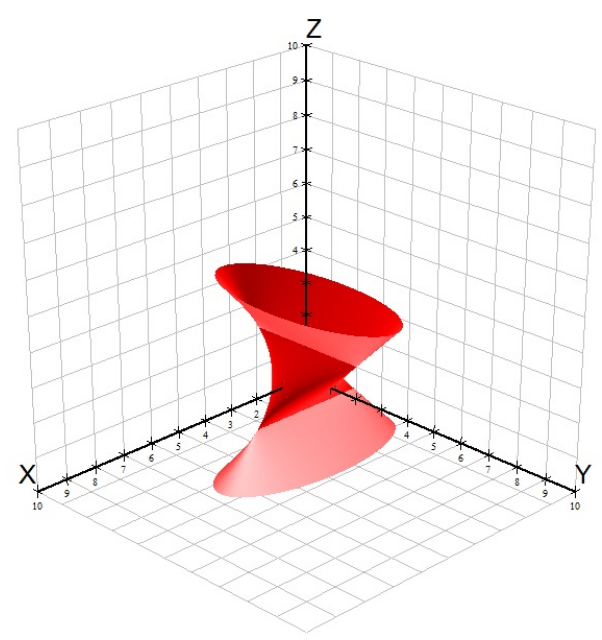

Fig. 1b. The normal developable surface

The Darboux vector fields which belong to this surface are found as:

$$
\begin{aligned}
& e_{1}=\left(-\frac{1}{\sqrt{2}} \sin s, \frac{1}{\sqrt{2}} \cos s, \frac{1}{\sqrt{2}}\right), \\
& e_{2}=\left(-\frac{1}{\sqrt{2}} \sin s, \frac{1}{\sqrt{2}} \cos s,-\frac{1}{\sqrt{2}}\right), \\
& e_{3}=(-\cos s,-\sin s, 0) .
\end{aligned}
$$

The directrix curve $\beta$ of the normal developable surface is in the following form:

$$
\beta=\int_{0}^{s} e_{3} d s=(-\sin s, \cos s, 0) .
$$

The geodesic curvature and geodesic torsion of the base curve are, respectively, computed as:

$$
\kappa_{g}=0, \quad \text { and } \quad \tau_{g}=\frac{1}{\sqrt{2}} .
$$

Then the ruling line $\tilde{\mathbf{e}}$ of the normal developable surface is obtained

$$
\tilde{\mathbf{e}}=\left(-\frac{1}{2} \sin s, \frac{1}{2} \cos s, \frac{1}{2}\right) \text {. }
$$

As a result, the normal developable surface $M_{n}$ is given with the below parameterization:

$$
\widetilde{\mathbf{P}}(s, v)=\left(-\sin s-\frac{v}{2} \sin s, \cos s+\frac{v}{2} \cos s, \frac{v}{2}\right) .
$$

The base surface given by (33) and the normal developable surface given by (34) have been together plotted in Fig. 1a. The normal developable surface given by (34) has been alone illustrated in Fig 1b. The normal developable surface has been illustrated by reflecting surface in Fig. 2. 


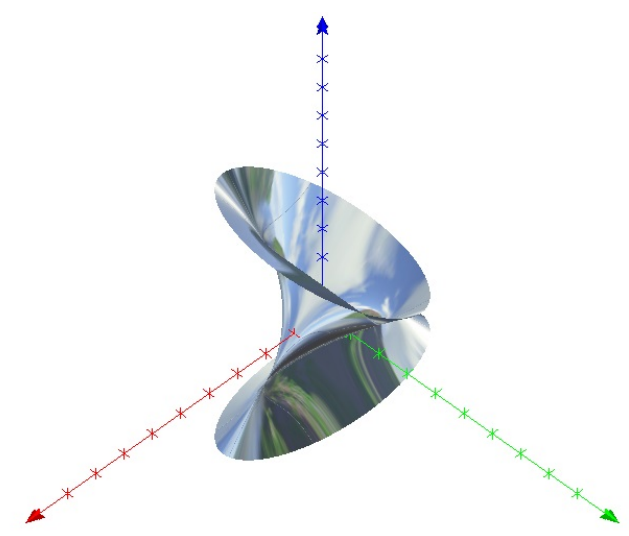

Fig. 2. The normal developable surface

Example 3.8. Let the base surface $M$ be given as the following parameterization:

$$
\mathbf{P}(s, v)=\left(\cos s-\frac{1}{\sqrt{2}} v \cos s, \sin s-\frac{1}{\sqrt{2}} v \sin s, \frac{v}{\sqrt{2}}\right) .
$$

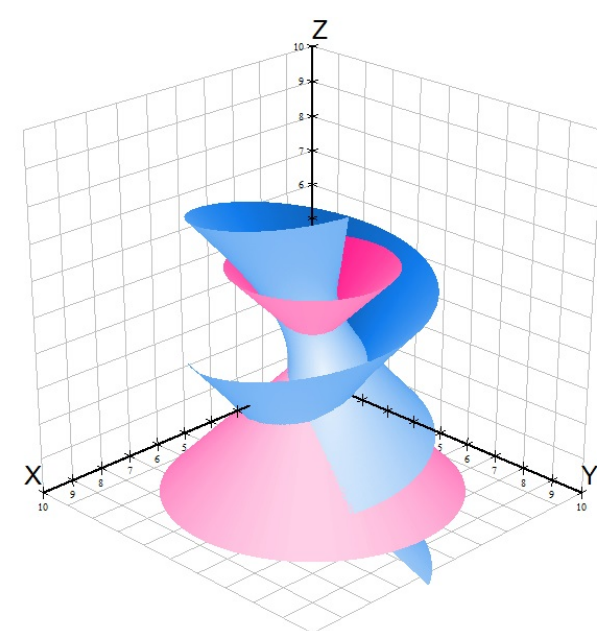

Fig. 3a. The base surface and its normal developable surface

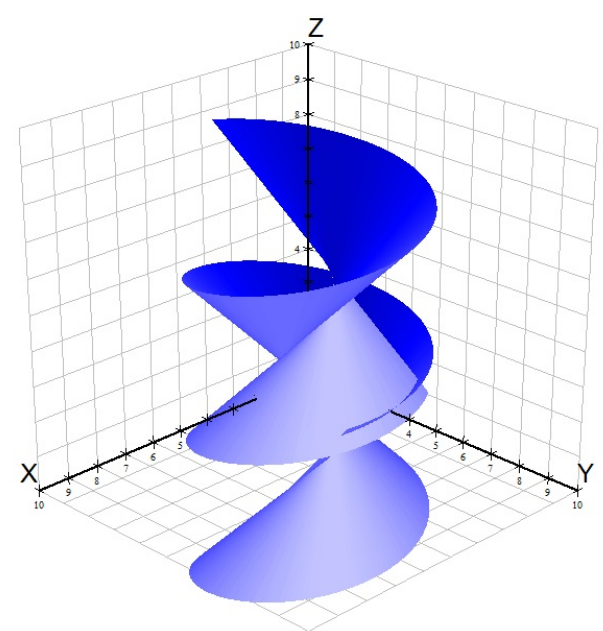

Fig. 3b. The normal developable surface

The Darboux vector fields which belong to this surface are found as:

$$
\begin{aligned}
& e_{1}=(-\sin s, \cos s, 0), \\
& e_{2}=\left(-\frac{1}{\sqrt{2}} \cos s,-\frac{1}{\sqrt{2}} \sin s, \frac{1}{\sqrt{2}}\right), \\
& e_{3}=\left(\frac{1}{\sqrt{2}} \cos s, \frac{1}{\sqrt{2}} \sin s, \frac{1}{\sqrt{2}}\right) .
\end{aligned}
$$


The directrix curve $\beta$ of the normal developable surface is in the following form:

$$
\begin{aligned}
\beta & =\int_{0}^{s} e_{3} d s \\
& =\left(\frac{1}{\sqrt{2}} \sin s,-\frac{1}{\sqrt{2}} \cos s, \frac{s}{\sqrt{2}}\right) .
\end{aligned}
$$

The geodesic curvature and geodesic torsion of the base curve are, respectively, computed as:

$$
\kappa_{g}=\frac{1}{\sqrt{2}}, \quad \text { and } \quad \tau_{g}=0
$$

Then the ruling line $\tilde{\mathbf{e}}$ of the normal developable surface is obtained

$$
\tilde{\mathbf{e}}=\left(\frac{1}{\sqrt{2}} \cos s, \frac{1}{\sqrt{2}} \sin s, \frac{1}{\sqrt{2}}\right) .
$$

As a result, the normal developable surface $M_{n}$ is given with the below parameterization:

$$
\widetilde{\mathbf{P}}(s, v)=\left(\frac{1}{\sqrt{2}} \sin s+\frac{1}{\sqrt{2}} v \cos s,-\frac{1}{\sqrt{2}} \cos s+\frac{1}{\sqrt{2}} v \sin s, \frac{s}{\sqrt{2}}+\frac{v}{\sqrt{2}}\right) .
$$

The base surface given by (35) and the normal developable surface given by (36) have been together plotted in Fig. 3a. The normal developable surface given by (36) has been alone illustrated in Fig 3b. The normal developable surface has been illustrated by reflecting surface in Fig. 4.

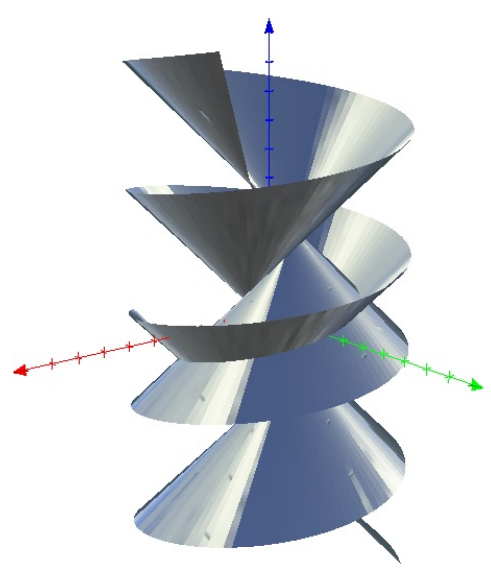

Fig. 4. The normal developable surface

Example 3.9. Let the base surface $M$ be given as the following parameterization:

$$
\mathbf{P}(s, v)=\left(\cos \frac{s}{\sqrt{2}}-\frac{1}{\sqrt{2}} v \sin \frac{s}{\sqrt{2}}, \sin \frac{s}{\sqrt{2}}+\frac{1}{\sqrt{2}} v \cos \frac{s}{\sqrt{2}}, \frac{s}{\sqrt{2}}+\frac{v}{\sqrt{2}}\right) .
$$




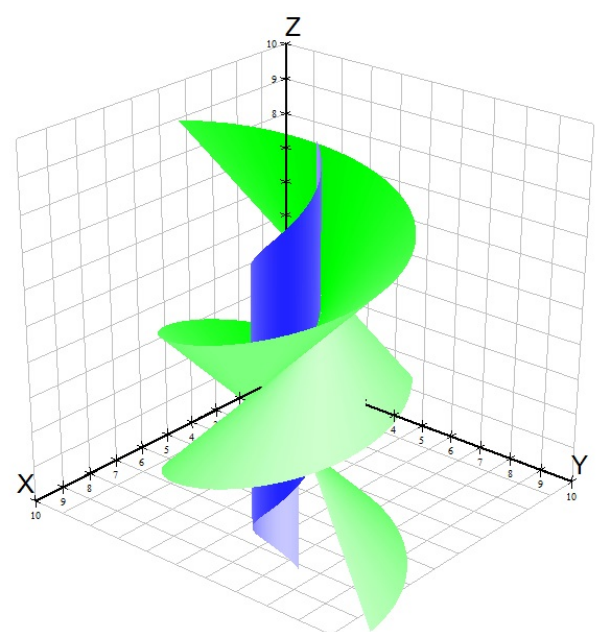

Fig. 5a. The base surface and its normal developable surface

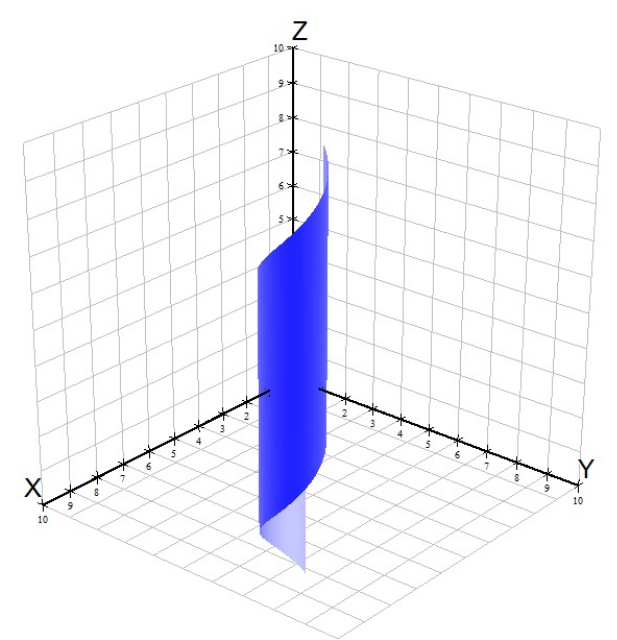

Fig. 5b. The normal developable surface

The Darboux vector fields which belong to this surface are found as:

$$
\begin{aligned}
& e_{1}=\left(-\frac{1}{\sqrt{2}} \sin \frac{s}{\sqrt{2}}, \frac{1}{\sqrt{2}} \cos \frac{s}{\sqrt{2}}, \frac{1}{\sqrt{2}}\right), \\
& e_{2}=\left(\cos \frac{s}{\sqrt{2}}, \sin \frac{s}{\sqrt{2}}, 0\right), \\
& e_{3}=\left(-\frac{1}{\sqrt{2}} \sin \frac{s}{\sqrt{2}}, \frac{1}{\sqrt{2}} \cos \frac{s}{\sqrt{2}},-\frac{1}{\sqrt{2}}\right) .
\end{aligned}
$$

The directrix curve $\beta$ of the normal developable surface is in the following form:

$$
\begin{aligned}
\beta & =\int_{0}^{s} e_{3} d s \\
& =\left(\cos \frac{s}{\sqrt{2}},-\sin \frac{s}{\sqrt{2}},-\frac{s}{\sqrt{2}}\right) .
\end{aligned}
$$

The geodesic curvature and geodesic torsion of the base curve are, respectively, computed as:

$$
\kappa_{g}=-\frac{1}{2}, \quad \text { and } \quad \tau_{g}=\frac{1}{2} .
$$

Then the ruling line $\tilde{\mathbf{e}}$ of the normal developable surface is obtained

$$
\tilde{\mathbf{e}}=\left(0,0, \frac{1}{\sqrt{2}}\right) .
$$

As a result, the normal developable surface $M_{n}$ is given with the below parameterization:

$$
\widetilde{\mathbf{P}}(s, v)=\left(\cos \frac{s}{\sqrt{2}},-\sin \frac{s}{\sqrt{2}},-\frac{s}{\sqrt{2}}+\frac{v}{\sqrt{2}}\right) .
$$

The base surface given by (37) and the normal developable surface given by (38) have been together plotted in Fig. 5a. The normal developable surface given by 
(38) has been alone illustrated in Fig 5b. The normal developable surface has been illustrated by reflecting surface in Fig. 6 .

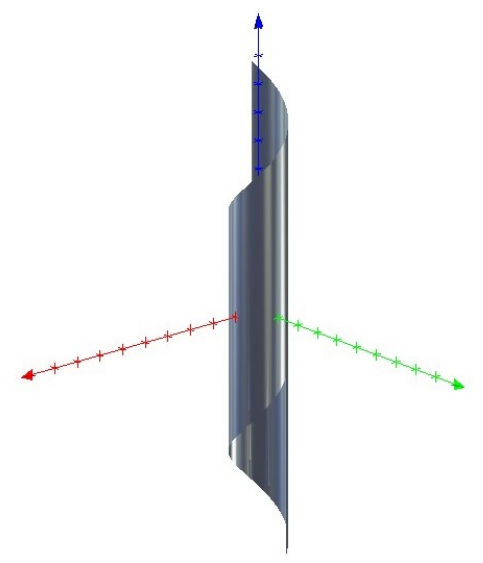

Fig. 6. The normal developable surface

\section{CONCLUDING REMARKS}

In this work, we examined normal developable surface of a surface along a normal direction curve $\beta$. We obtained some results of these kind surfaces. The methodology used in [7], [8] and here can be applied to the pair "curve and ruled surface" in different spaces such as Lorentz-Minkowski space, isotropic space, and etc. Also choosing the directrix curve of ruled surface as different direction or donor curves, different studies can be made for curves on ruled surfaces.

Acknowledgement. The authors would like to thank the anonymous reviewers for their valuable comments and suggestions to improve the quality of the paper.

\section{REFERENCES}

[1] E. Abbena, S. Salamon, Alfred Gray, Modern differential geometry of curves and surfaces with Mathematica, Chapman and Hall/CRC; 3 edition (June 21, 2006).

[2] R. Cipolla, P. J. Giblin, Visual Motion of Curves and Surfaces, Cambridge Univ. Press, 2000.

[3] F. Hathout, M. Bekar, and Y. Yayl, Ruled surfaces and tangent bundle of unit 2-sphere, International Journal of Geometric Methods in Modern Physics, 14(10) (2017),1750145.

[4] S. Izumiya and N. Takeuchi, Geometry of Ruled Surfaces, Applicable Mathematics in the Goldon Age (ed. J. C. Misra), 305-308, Narosa Pulishing House, New Delhi, 2003.

[5] S. Izumiya, N. Takeuchi, Special curves and ruled surfaces, Beitr. Algebra Geom. 44(1) (2003), 203-212.

[6] S. Izumiya, N. Takeuchi, New special curves and developable surfaces, Turk J Math, 28(2) (2004), 153-163.

[7] S. Izumiya and S. Otani, Flat approximations of surfaces along curves, Demonstr. Math. 48(2) (2015) 1-7. 
[8] S. Hananoi, S. Izumiya, Normal developable surfaces of surfaces along curves. Proceedings of the Royal Society of Edinburgh: Section A Mathematics, 147(1) (2017), 177-203.

[9] I. Markina, M. Raffaelli, Flat approximations of hypersurfaces along curves, manuscripta math. (2018). https://doi.org/10.1007/s00229-018-1072-6.

[10] R. S. Milman, and G. D. Parker, Elements of Differential Geometry, Prentice-Hall Inc., Englewood Cliffs, New Jersey, (1977).

[11] I. R. Porteous, Geometric Differentiation for the Intelligence of Curves and Surfaces (second edition). Cambridge University Press, Cambridge, (2001).

[12] I. Vaisman, A First Course in Differential Geometry, Pure and Applied Mathematics, A Series of Monograph and Textbooks, Marcel Dekker, (1984). 